This item was submitted to Loughborough's Research Repository by the author.

Items in Figshare are protected by copyright, with all rights reserved, unless otherwise indicated.

\title{
Ab-initio modelling of defects in $\mathrm{MgO}$
}

PLEASE CITE THE PUBLISHED VERSION

http://dx.doi.org/10.1016/j.nimb.2006.11.044

PUBLISHER

(c) Elsevier

VERSION

AM (Accepted Manuscript)

LICENCE

CC BY-NC-ND 4.0

REPOSITORY RECORD

Gilbert, Christopher A., Roger Smith, and Steven D. Kenny. 2019. "Ab-initio Modelling of Defects in Mgo". figshare. https://hdl.handle.net/2134/11639. 
This item was submitted to Loughborough's Institutional Repository (https://dspace.lboro.ac.uk/) by the author and is made available under the following Creative Commons Licence conditions.

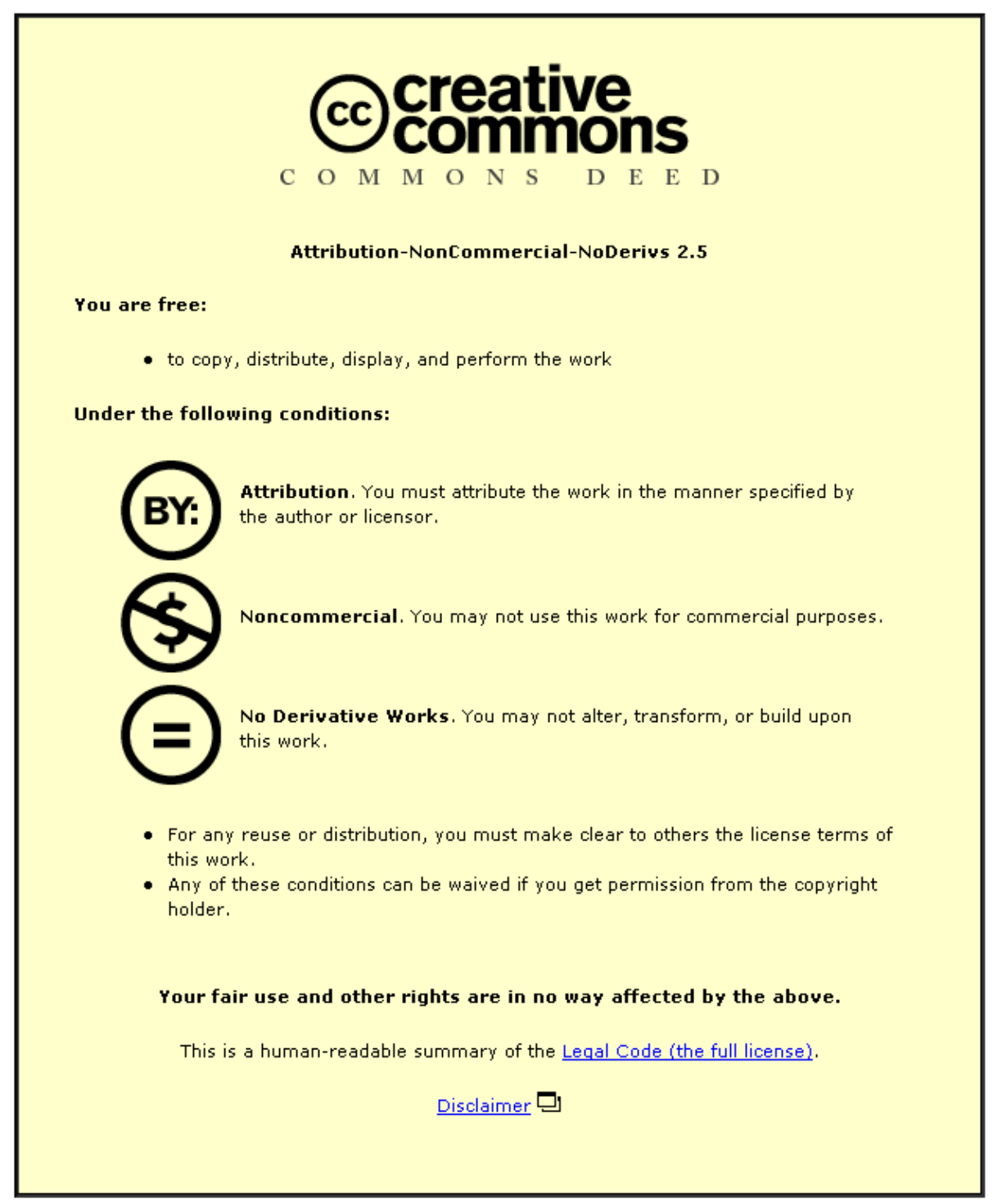

For the full text of this licence, please go to: http://creativecommons.org/licenses/by-nc-nd/2.5/ 


\title{
Ab-initio modelling of defects in $\mathrm{MgO}$
}

\author{
C.A. Gilbert R. Smith S.D. Kenny* \\ Department of Mathematical Sciences, Loughborough University, Loughborough, \\ Leicestershire, LE11 3TU, UK.
}

\begin{abstract}
The energetics of the key defects that are observed to occur during simulations of radiation damage in $\mathrm{MgO}$ are analysed using density functional theory. The results are compared with those from the empirical potentials used to carry out the radiation damage studies. The formation energies of vacancies, interstitials, Frenkel pairs, di-vacancies and di-interstitials are calculated as a function of the increasing supercell size in order to ensure good convergence. The supercell geometries were chosen to maximise the separation distance between periodic images. Their sizes ranged from cells containing 32 atoms up to cells containing 180 atoms.

Results are presented for the formation energies of the first, second and third nearest neighbour defects. Results show that the di-vacancy formation energy is in the region of 4-6 eV and that formation energies for di-interstitials are more than double this, lying in the range $12-16 \mathrm{eV}$. Comparison of the results show that empirical potentials overestimate the formation energy of di-vacancies by $1-3 \mathrm{eV}$ and underestimate the formation energies of di-interstitials by about $1-2 \mathrm{eV}$. The relative stability of the defects is, however, correctly predicted by the empirical potentials. The direction and the magnitude of the displacements of the atoms surrounding the defects are in good agreement for all the systems containing interstitials. For the systems containing vacancies the direction of the displacements are in agreement but the empirical potentials predict larger displacements in all cases.
\end{abstract}

Key words: Magnesium oxide, Defects

PACS: 31.13.Ew, 61.72.-y, 61.82.Ms

* Corresponding author

Email address: S.D.Kenny@lboro.ac.uk (S.D. Kenny).

URL: http://www-staff.lboro.ac.uk/ masdk (S.D. Kenny). 


\section{Introduction}

Over the years, much attention has been given to the study of radiation damage of materials. Materials such as ceramics based on the $\mathrm{MgO}-\mathrm{Al}_{2} \mathrm{O}_{3}$ system are the subject of such analysis due to the fact that they are of interest as materials for long term storage of radioactive substances, such as nuclear waste, which needs to be stored safely for long periods of time. Magnesium oxide is the simplest representative for a large class of such oxides. The fact that $\mathrm{MgO}$ has the $\mathrm{NaCl}$ or rocksalt structure makes $\mathrm{MgO}$ much more manageable to study than more complex oxides. $\mathrm{MgO}$ is a two component oxide with a relatively simple crystal structure and has a strong ionic nature. It has well understood properties and as a result has often been utilised as a test material upon which to perform both standard experiments and computer simulations. Many molecular dynamics simulations investigating the behaviour of $\mathrm{MgO}$ have been performed $[1,2]$ and there are well established empirical potentials that exist for it.

In order to study radiation damage in $\mathrm{MgO}$, energetics of defects should be examined, as this will provide a useful insight into the general behaviour of ionic materials when subjected to radiation damage. Previous molecular dynamics simulations $[1,2]$ have proven to be very useful in that they provide information about the different types of defects created when a material is subjected to such damage. The main body of this work is concerned with the study of energetics of isolated Frenkel pairs, di-vacancies and di-interstitials, since it is these that were shown to appear most frequently in cascade simulations in $\mathrm{MgO}$. The majority of the studies of the defect energetics in this material have been based on empirical potential models. These assume pair potentials between ions in the system and often use the shell model [3-5]. As successful as these models have been, there remains doubts about the accuracy of such methodologies in the prediction of defect energies. Thus in this work we have studied the energetics of a number of defects using a DFT approach.

There has only been a small amount of previous work carried out using abinitio methods to study defect energetics in $\mathrm{MgO}$. De Vita et. al [6,7] calculated the formation energy of a pair of isolated vacancies using DFT. Their results showed that the formation energy is expected to lie somewhere between 6 and $7 \mathrm{eV}$. This conclusion was reached using a fairly small supercell consisting of 32 atoms. In much more recent work by Alfe and Gillan [8] results of DFT calculations of pairs of isolated vacancies show a similar result and are also shown to be in good agreement with quantum Monte Carlo calculations. In that the formation energies of isolated vacancies are found to be between 6 and $7.5 \mathrm{eV}$. In this work we have studied not just isolated vacancies but also systems containing interstitials and vacancies and interstitials that neighbour each other. 


\section{Methodology}

The ab initio calculations were performed using a localised orbital based density functional theory (DFT) [9,10] code, Plato [11]. The pseudopotentials of Hartwigsen, Goedecker and Hutter [12] were employed to describe the electron-ion interactions, with a semi-core pseudopotential used for $\mathrm{Mg}$. The local-density approximation (LDA) [13] to the exchange correlation potential was used. A triple numeric double polarisation basis set containing 14 basis functions for $\mathrm{Mg}$ and 23 basis functions for $\mathrm{O}$ was employed. A $6 \times 6 \times 6$ Monkhorst-Pack [14] k-point mesh was used for the unit cell; with equivalent meshes being employed for the larger cells. Using this approach we get an equilibrium lattice constant of $4.17 \AA$ and a bulk modulus of $1.65 \mathrm{Mbar}$. These compare well with the earlier results of De Vita et. al [7] of $4.17 \AA$ and 1.54 Mbar.

MD simulations of radiation damage have shown that the most common types of defect found in $\mathrm{MgO}$ after a simulation are isolated Frenkel pairs, di-vacancies and di-interstitials. In this work, we have studied the formation energy of such defects as a function of the separation distance of the individual vacancies or interstitials that constitute the defect pair. We have also calculated the formation energy of a pair of isolated vacancies, interstitials and of isolated Frenkel pairs. The defects have been labelled throughout this work to make them easier to refer to. The isolated defect pairs are labelled "Defect 0". The di-vacancies are labelled "Defect 1", "Defect 2" and "Defect 3" corresponding to whether the defects are in first, second or third nearest neighbour positions, taking into account the fact that a di-vacancy here consists of a removal of both an $\mathrm{Mg}$ and an $\mathrm{O}$ atom. Di-interstitals are labelled in a similar manner, and such that the relative positions of atoms for each of the defects are identical to that of their di-vacancy counterparts; for di-vacancies these are in the first, third and fifth nearest neighbour positions.

The calculations using DFT have employed periodic boundary conditions and thus a supercell approach has been taken. In order to minimise the effect of the interaction between defects and their periodic repeats we have studied the defect energies as a function of supercell size. To enable this, a methodology was implemented that allowed a variety of supercell shapes to be created. The method is also implemented in the computational tool CRYSTAL [15]. A supercell is created by using a transformation matrix $T$ where $t_{i j}$ are integers for $i, j=1 \ldots 3$. This is then used to construct three supercell vectors $\left(\mathbf{s}_{\mathbf{1}}, \mathbf{s}_{\mathbf{2}}, \mathbf{s}_{\mathbf{3}}\right)$ 
from the primitive cell vectors $\left(\mathbf{p}_{\mathbf{1}}, \mathbf{p}_{\mathbf{2}}, \mathbf{p}_{\mathbf{3}}\right)$.

$$
\begin{aligned}
& T=\left(\begin{array}{ccc}
t_{11} & t_{12} & t_{13} \\
t_{21} & t_{22} & t_{23} \\
t_{31} & t_{32} & t_{33}
\end{array}\right), \\
& \mathbf{s}_{\mathbf{1}}=t_{11} \mathbf{p}_{\mathbf{1}}+t_{12} \mathbf{p}_{\mathbf{2}}+t_{13} \mathbf{p}_{\mathbf{3}} \\
& \mathbf{s}_{\mathbf{2}}=t_{21} \mathbf{p}_{\mathbf{1}}+t_{22} \mathbf{p}_{\mathbf{2}}+t_{23} \mathbf{p}_{\mathbf{3}} \\
& \mathbf{s}_{\mathbf{3}}=t_{31} \mathbf{p}_{\mathbf{1}}+t_{32} \mathbf{p}_{\mathbf{2}}+t_{33} \mathbf{p}_{\mathbf{3}}
\end{aligned}
$$

In order to optimise the simulations we have minimised the number of atoms in the supercell whilst holding the minimum separation between the defect and its periodic repeats fixed. Using this we have constructed the twelve supercells that are used in the DFT calculations on the defect pairs. These are given in table 1 .

For a pair of isolated point defects, the formation energy is computed using equation (3). Here, $E_{\text {form }}$ is the required formation energy, $n$ is the total number of lattice sites in the supercell and $E_{n}(i, j)$ is the energy of a relaxed supercell with $n$ lattice sites containing $i$ defects of one species and $j$ defects (of the same type) of the other species. The \pm is positive in the case of a pair of isolated interstitials and negative for a pair of isolated vacancies.

$$
E_{\text {form }}=E_{n}(1,0)+E_{n}(0,1)-2\left(\frac{n \pm 1}{n}\right) E_{n}(0,0)
$$

For a supercell containing a pair of interstitials or vacancies this is simplified to equation (4).

$$
E_{\text {form }}=E_{n}(1,1)-\left(\frac{n \pm 2}{n}\right) E_{n}(0,0)
$$

The DFT results have been compared with results acquired using empirical potentials. The empirical potential results were obtained using a fixed charge model, with charges of +2 on the $\mathrm{Mg}$ ions and -2 on the $\mathrm{O}$ ions. The Buckingham potential (without shell) was used to describe the short-range interactions to be consistent with simulations carried out in [1]. The potential for this is given in equation (5); with the parameters $A, \rho, C$ and $r_{1}$ taking the values given in [2].

$$
\phi(r)=A \exp \left(-\frac{r}{\rho}\right)-\frac{C}{r^{6}}+V(r)
$$


For the empirical potential calculations cubic cells containing at least 64,000 ions in conjunction with periodic boundary conditions were employed.

\section{Results}

The formation energies from the DFT calculations for the pairs of isolated vacancies and interstitials as a function of system size are given in table 2 . The formation energy for the isolated vacancies is $\approx 5.9 \mathrm{eV}$ whereas the formation energy for the di-interstitial is $\approx 16.6 \mathrm{eV}$. Calculations performed using empirical potentials gave a formation energy for the isolated vacancies of 8.8 $\mathrm{eV}$ with a formation energy for the di-interstitial of $18.4 \mathrm{eV}$. These formation energies are larger than the ones calculated from the DFT simulations, which is a trend that is common throughout all the defect energies calculated. The formation energies from the DFT calculations for the isolated $\mathrm{Mg}$ and O Frenkel pairs as a function of system size are also given in table 2 . The formation energy of the isolated $\mathrm{Mg}$ Frenkel pair is $\approx 10.3 \mathrm{eV}$ whereas the formation energy for the isolated $\mathrm{O}$ Frenkel pair is $\approx 12.2 \mathrm{eV}$. This compares to empirical potential results of $14.1 \mathrm{eV}$ and 13.6 for the isolated $\mathrm{Mg}$ and $\mathrm{O}$ Frenkel pairs respectively.

Figures 1 and 2 show how the defect energies from the DFT calculations for the di-vacancies and di-interstitials respectively converge as a function of the separation distance between the defects and their periodic repeats. It can be seen that the defect energies are converged to within $\pm 0.2 \mathrm{eV}$. These formation energies computed using DFT are compared to the formation energies for the same defects obtained using empirical potentials in table 3. The DFT results in the table are the results using the largest supercell considered for that defect.

In addition to comparing the formation energies calculated using the two methods, a comparison has also been made between the resulting relaxed structures of the atoms surrounding the defects. Figure 3 shows the directions of the relaxations of the atoms surrounding the pairs of isolated defects, with table 4 giving the magnitude of the relaxations. The black circles in the diagrams are $\mathrm{Mg}$ atoms, the white circles $\mathrm{O}$ atoms, with the squares representing vacancies. The vacancies attract neighbouring atoms of the same species along the common line of action whilst repelling the other species of atom. The interstitials behave in the opposite fashion. In the case of the interstitials the displacement of atoms of the same species is much larger than the displacement of the other species. Looking at the DFT results for the $\mathrm{Mg}$ interstitial the surrounding $\mathrm{Mg}$ atoms are displaced by $0.45 \AA$ compared to $0.023 \AA$ for the surrounding $\mathrm{O}$ atoms and for the $\mathrm{O}$ interstitial the surrounding $\mathrm{O}$ atoms are displaced by $0.48 \AA$ compared to $0.017 \AA$ for the surrounding $\mathrm{Mg}$ atoms. 
The results from the empirical potential calculations show a similar pattern to those from the DFT calculations for the relaxation around the isolated vacancies, but the magnitude of the displacements in the empirical potential case is about double that of the DFT case. For the case of the isolated interstitials the displacement of the like species follows a similar pattern to the DFT case and the magnitudes of the displacements are very similar. The displacement of the other species for the case of the isolated vacancies is, however, in the opposite direction; with the $\mathrm{O}$ ion moving towards the $\mathrm{Mg}$ interstitial and the $\mathrm{Mg}$ ion moving towards the $\mathrm{O}$ interstitial. The magnitudes of the displacements of the other species in both the DFT and the empirical potential simulations are very small, being less than $0.025 \AA$. Thus this is a small error which means that the relaxation of the atoms around the interstitials is found to be very similar for the DFT and empirical potential simulations.

The directions and magnitudes of the the displacements of the atoms surrounding first nearest neighbour di-vacancies and di-interstitials defects are given in figure 4 and 5 respectively. When relaxed the interstitials in the di-interstitial case move towards each other. These still do not form split interstitials but remain central in relation to the surrounding ions. The relaxations obtained by DFT and empirical potentials are again very similar for the di-interstitial case. For the case of the di-vacancy all the atom movements barring the $\mathrm{O}$ atoms surrounding the $\mathrm{Mg}$ vacancy are very similar. Displacements that are not labelled are less than $0.09 \AA$.

The directions and magnitudes of the the displacements of the atoms surrounding second nearest neighbour di-vacancies and di-interstitials defects are given in figure 5 and 6 respectively. A similar pattern is seen for the comparison of the results from the DFT and the empirical potential calculations, in that there is good agreement between the relaxations around the di-interstitial, with the direction of the displacements for the di-vacancies agreeing but the empirical potential results predicting a larger relaxation. The di-interstitial in this case is seen to form a crowdion along $<111>$, this is also observed for the third nearest neighbour di-interstitial case. Results for the third nearest neighbour defects show a very similar trend.

\section{Conclusions}

From the results it can be seen that the empirical potential simulations overestimate the formation energies for the di-vacancy by 1-3 eV. They underestimate the formation energies for the nearest neighbour di-interstitials by less than $1 \mathrm{eV}$, the isolated interstitial formation energy is, however, overestimated by about $2 \mathrm{eV}$. Both methods predict similar relaxed structures for the defects but the empirical potentials overestimate the amount by which some of the 
atoms surrounding the vacancies relax.

For the di-vacancies the first nearest neighbour di-vacancies configuration is predicted as being the most energetically favourable structure by both the DFT and empirical potential calculations. The DFT results predict that configurations where the di-vacancies have a larger separation are comparable in energy to the isolated defect pair. The empirical potential results imply that these structures are energetically more favourable than the isolated defect pair.

For the case of the di-interstitials the first and fifth neighbour cases are the most energetically favourable configurations according to both the empirical potential and the DFT calculations. The formation energy of the third nearest neighbour di-interstitial is much closer to that for the isolated interstitials.

\section{References}

[1] B. P. Uberuaga, R. Smith, A. R. Cleave, F. Montalenti, G. Henkelman, R. W. Grimes, A. F. Voter, K. E. Sickafus, Structure and mobility of defects formed from collision cascades in mgo, Phys. Rev. Lett. 92 (11) (2004) 115505.

[2] B. P. Uberuaga, R. Smith, A. R. Cleave, G. Henkelman, R. W. Grimes, A. F. Voter, K. E. Sickafus, Dynamical simulations of radiation damage and defect mobility in mgo, Phys. Rev. B 71 (2005) 104102.

[3] N. F. Mott, M. J. Littleton, Trans. Faraday Soc. 34 (1938) 485.

[4] C. R. A. Catlow, W. C. Mackrodt, Computer Simulation of Solids, SpringerVerlag, 1982.

[5] J. H. Harding, Computer simulation of defects in ionic solids, Rep. Prog. Phys. 53 (1990) 1403-1466.

[6] A. D. Vita, M. J. Gillan, J. S. Lin, M. C. Payne, I. Stich, L. J. Clarke, Defect energetics in oxide materials from first principles, Phys. Rev. Lett. 68 (22) (1992) 3319-3322.

[7] A. D. Vita, M. J. Gillan, J. S. Lin, M. C. Payne, I. Stich, L. J. Clarke, Defect energetics in mgo treated by first-principles methods, Phys. Rev. B 46 (20) (1992) 12964-12973.

[8] D. Alfe, M. J. Gillan, Schottky defect formation energy in mgo calculated by diffusion monte carlo, Phys. Rev. B 71 (2005) 220101.

[9] P. Hohenberg, W. Kohn, Inhomogeneous electron gas, Phys. Rev. 136 (3B) (1964) B864-B871.

[10] W. Kohn, L. J. Sham, Self-consistent equations including exchange and correlation effects, Phys. Rev. 140 (4A) (1965) A1133-A1138. 
[11] S. D. Kenny, A. P. Horsfield, H. Fujitani, Transferable atomic-type orbital basis sets for solids, Phys. Rev. B 62 (8) (2000) 4899-4905.

[12] C. Hartwigsen, S. Goedecker, J. Hutter, Relativistic separable dual-space gaussian pseudopotentials from h to rn, Phys. Rev. B 58 (7) (1998) 3641-3662.

[13] S. Goedecker, M. Teter, J. Hutter, Separable dual-space gaussian pseudopotentials, Phys. Rev. B 54 (1996) 1703-1710.

[14] H. J. Monkhorst, J. D. Pack, Special points for brillouin-zone integrations, Phys. Rev. B 13 (1976) 51885192.

[15] V. R. Saunders, R. Dovesi, C. Roetti, R. Orlando, C. M. Zicovich-Wilson, N. M. Harrison, K. Doll, B. Civalleri, I. Bush, P. D'Arco, M. Llunell, CRYSTAL2003 User's Manual, University of Torino, 2003.

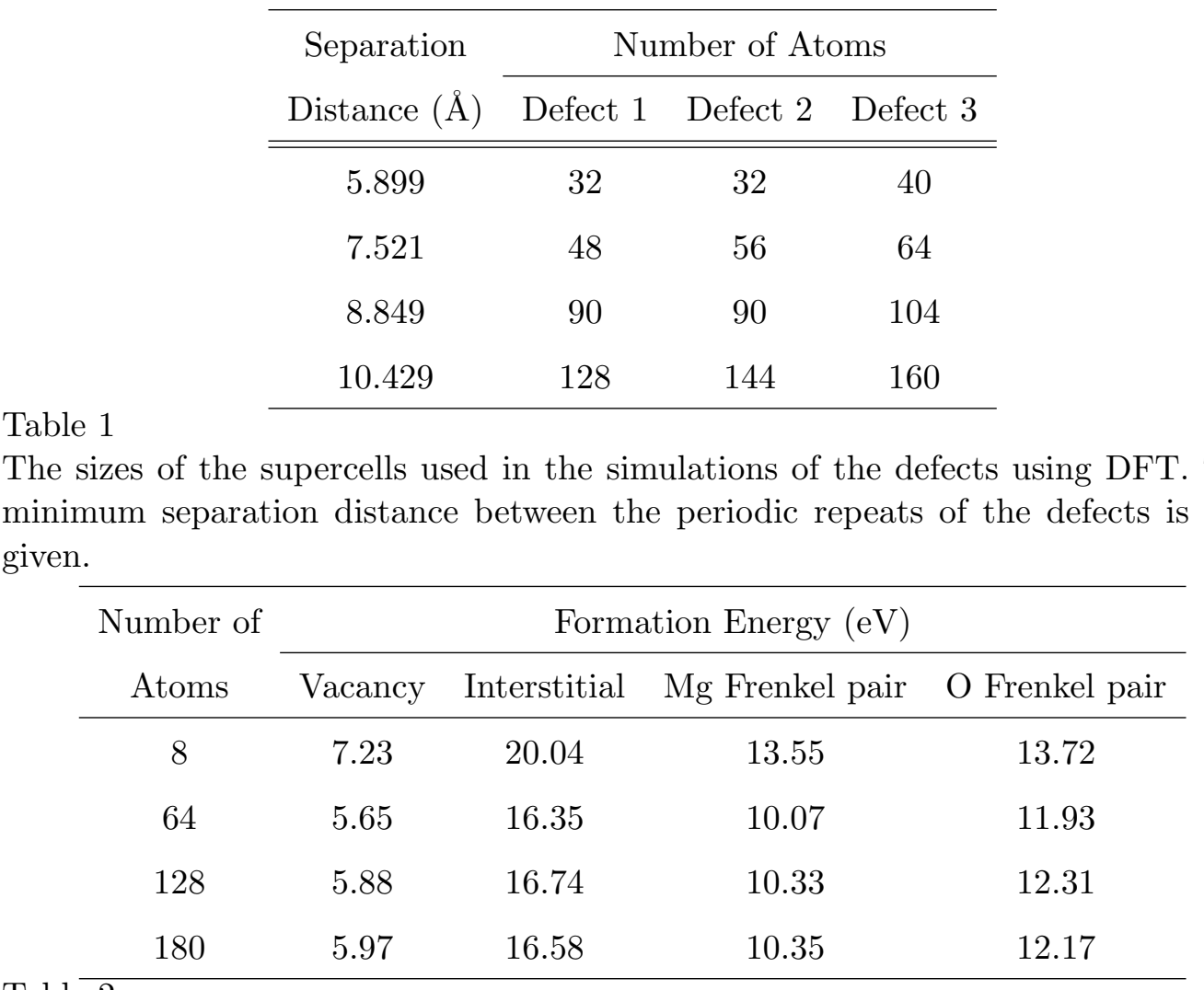

Table 1

Table 2

The formation energies from the DFT calculations for the pairs of isolated defects as a function of the system size. 


\begin{tabular}{ccccc}
\hline \multirow{2}{*}{ Defect } & \multicolumn{2}{c}{ DFT Formation Energy $(\mathrm{eV})$} & Empirical Potential Formation Energy $(\mathrm{eV})$ \\
\cline { 2 - 5 } & Di-vacancy & Di-interstitial & Di-vacancy & Di-interstitial \\
\hline 1 & 4.6 & 12.4 & 5.7 & 11.6 \\
2 & 5.9 & 15.4 & 7.7 & 15.3 \\
3 & 5.7 & 12.1 & 7.2 & 11.5 \\
\hline
\end{tabular}

Table 3

The defect formation energies for the nearest neighbour di-vacancies and diinterstitials for both the DFT and the empirical potential calculations as a function of the defect separation.

\begin{tabular}{cccc}
\hline Defect & Label & DFT & $\begin{array}{c}\text { Empirical Potential } \\
\text { displacements }(\AA)\end{array}$ \\
\hline Mg vacancy & A & 0.08 & 0.14 \\
Mg vacancy & B & 0.12 & 0.21 \\
\hline O vacancy & C & 0.07 & 0.11 \\
O vacancy & D & 0.16 & 0.21 \\
\hline Mg interstitial & E & 0.023 & -0.023 \\
Mg interstitial & F & 0.45 & 0.49 \\
\hline O interstitial & G & 0.017 & -0.024 \\
O interstitial & H & 0.48 & 0.47 \\
\hline
\end{tabular}

Table 4

The magnitude of the displacements of the atoms during relaxation by the DFT and the empirical potential methods. The labels refer to the letters in figure 3 .

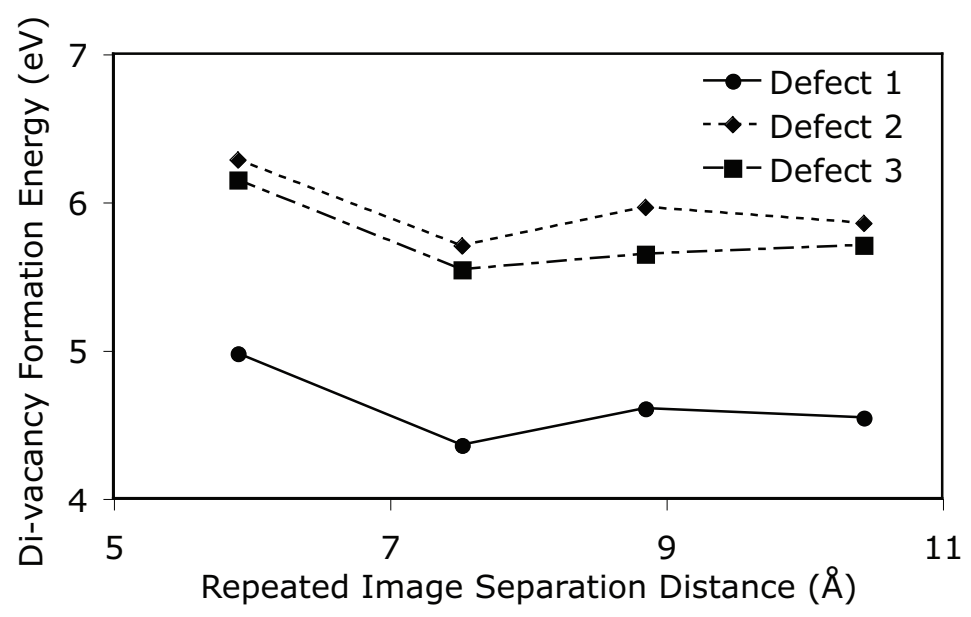

Fig. 1. The convergence of formation energies for di-vacancies as calculated by DFT as a function of the system size. 


\begin{tabular}{cccc}
\hline Defect & Label & $\begin{array}{c}\text { DFT } \\
\text { displacements }(\AA)\end{array}$ & $\begin{array}{c}\text { Empirical Potential } \\
\text { displacements }(\AA)\end{array}$ \\
\hline di-vacancy & A & 0.12 & 0.19 \\
di-vacancy & B & 0.17 & 0.18 \\
di-vacancy & C & 0.12 & 0.19 \\
di-vacancy & D & 0.16 & 0.18 \\
di-vacancy & E & 0.09 & 0.10 \\
di-vacancy & F & 0.09 & 0.10 \\
\hline di-interstitial & A & 0.13 & 0.13 \\
di-interstitial & B & 0.12 & 0.14 \\
di-interstitial & $\mathrm{C}$ & 0.42 & 0.43 \\
di-interstitial & D & 0.43 & 0.42 \\
di-interstitial & E & 0.58 & 0.54 \\
di-interstitial & F & 0.57 & 0.54 \\
\hline
\end{tabular}

Table 5

The magnitude of the displacements of the atoms during relaxation of the first nearest neighbour di-vacancy and di-interstitial defects by the DFT and the empirical potential methods. The labels refer to the letters in figure 4 .

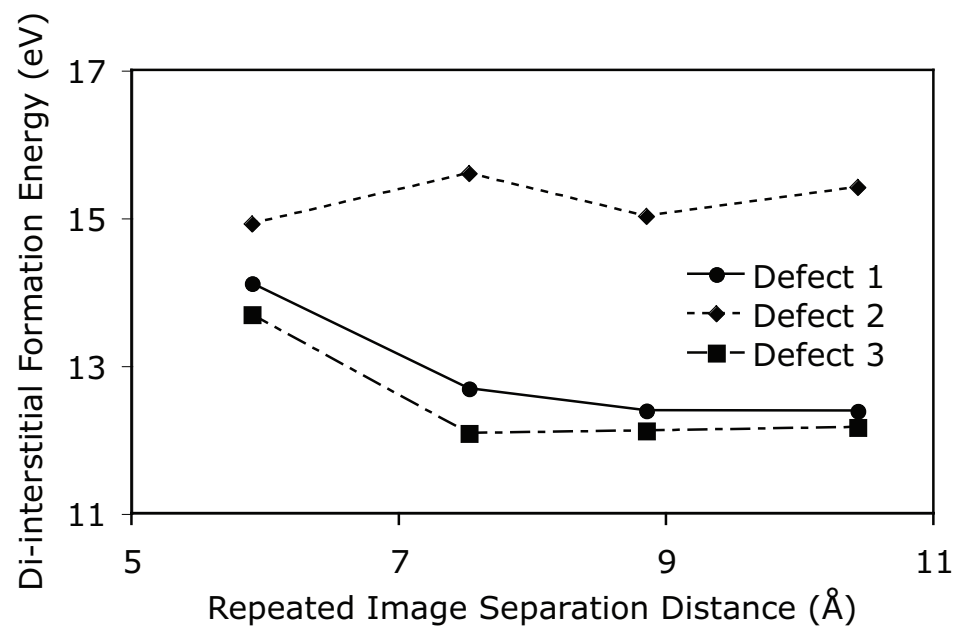

Fig. 2. The convergence of formation energies for di-interstitials as calculated by DFT as a function of the system size. 


\begin{tabular}{cccc}
\hline Defect & Label & $\begin{array}{c}\text { DFT } \\
\text { displacements }(\AA)\end{array}$ & $\begin{array}{c}\text { Empirical Potential } \\
\text { displacements }(\AA)\end{array}$ \\
\hline di-vacancy & $\mathrm{A}$ & 0.13 & 0.20 \\
di-vacancy & $\mathrm{B}$ & 0.17 & 0.19 \\
\hline di-interstitial & $\mathrm{A}$ & 0.88 & 0.89 \\
di-interstitial & $\mathrm{B}$ & 0.45 & 0.39 \\
di-interstitial & $\mathrm{C}$ & 1.37 & 1.38 \\
di-interstitial & $\mathrm{D}$ & 0.87 & 0.88 \\
di-interstitial & $\mathrm{E}$ & 0.22 & 0.21 \\
di-interstitial & $\mathrm{F}$ & 0.13 & 0.11 \\
di-interstitial & $\mathrm{G}$ & 0.37 & 0.36 \\
di-interstitial & $\mathrm{H}$ & 0.20 & 0.22 \\
di-interstitial & $\mathrm{I}$ & 0.21 & 0.25 \\
\hline
\end{tabular}

Table 6

The magnitude of the displacements of the atoms during relaxation of the second nearest neighbour di-vacancy and di-interstitial defects by the DFT and the empirical potential methods. The labels refer to the letters in figure 5 . 

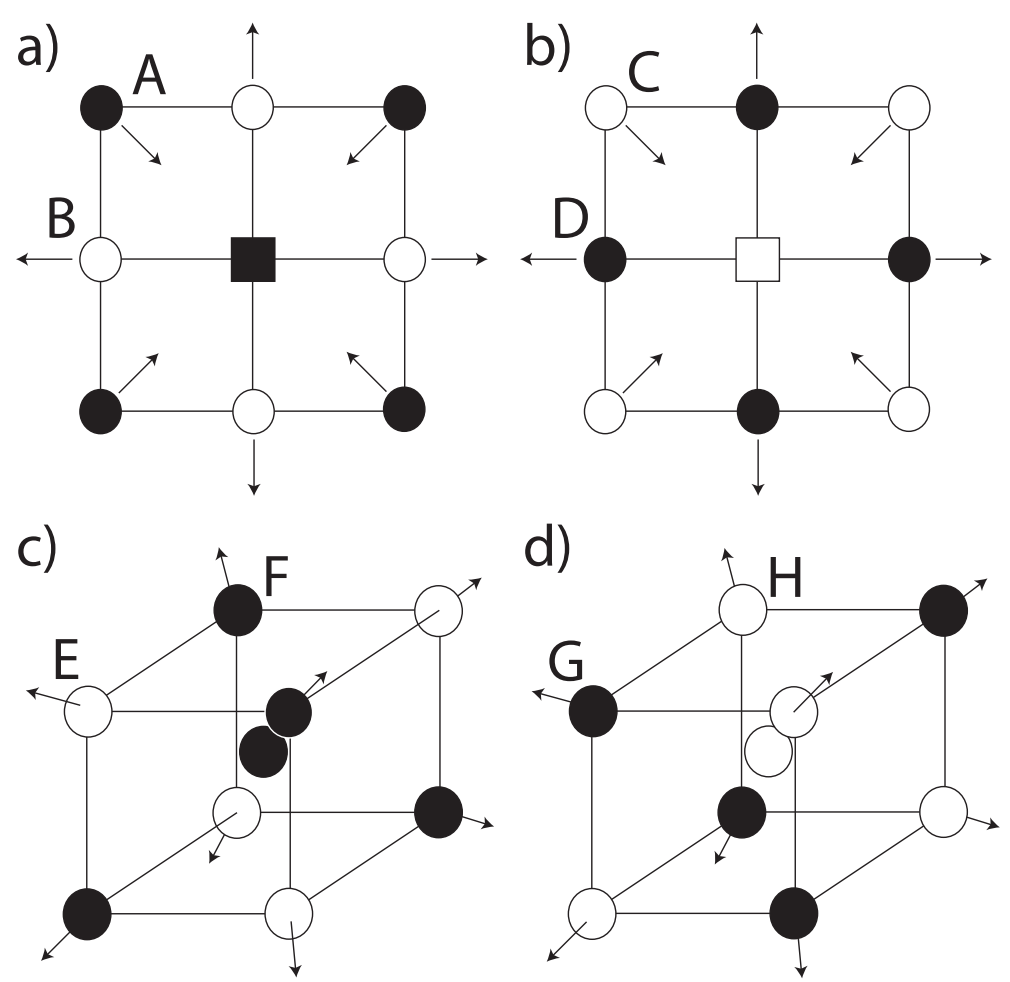

Fig. 3. The relaxed structure of the atoms surrounding the isolated defects, the magnitudes of the relaxations are given in table 4. (a) refers to the $\mathrm{Mg}$ vacancy, (b) to the $\mathrm{O}$ vacancy, (c) to the $\mathrm{Mg}$ interstitial and (d) to the $\mathrm{O}$ interstitial.

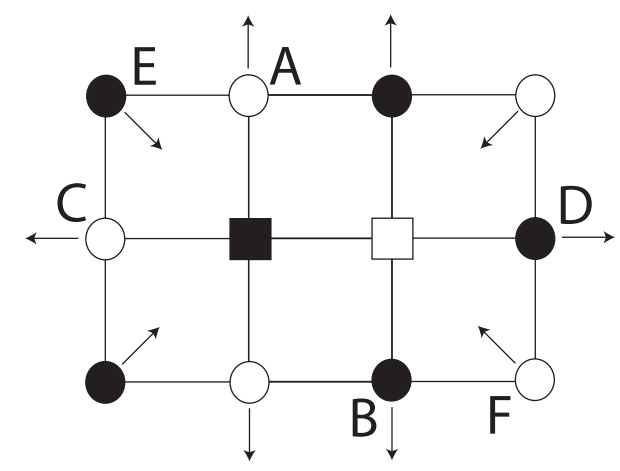

(a)

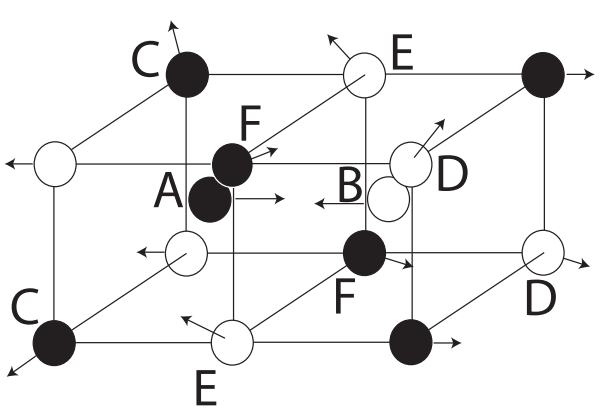

(b)

Fig. 4. The relaxed structure of the atoms surrounding the first nearest neighbour di-vacancies and di-interstitials, the magnitudes of the relaxations are given in table 5. (a) refers to the di-vacancy, (b) to the di-interstitial. 


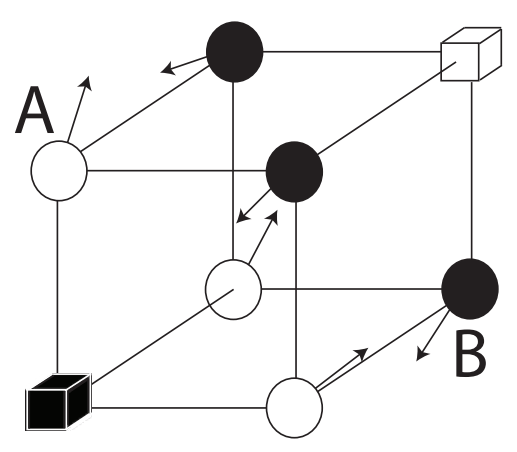

(a)

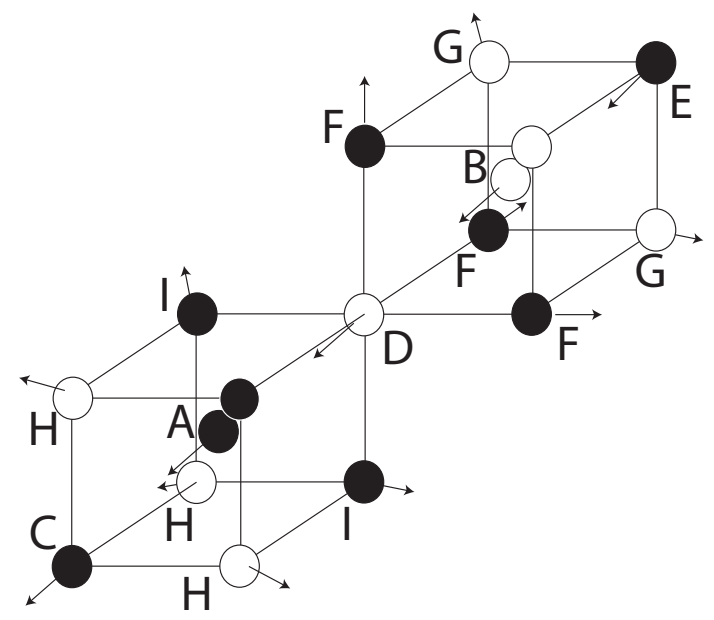

(b)

Fig. 5. The relaxed structure of the atoms surrounding the second nearest neighbour di-vacancies and di-interstitials, the magnitudes of the relaxations are given in table 6. (a) refers to the di-vacancy, (b) to the di-interstitial. 